\title{
THREE-DIMENSIONAL DENSITOMETRIC RECONSTRUCTION AND VISUALIZATION OF STENOSED CORONARY ARTERY SEGMENTS
}

\author{
J. G. M. van den Broek, ${ }^{*}$ C. H. Slump, ${ }^{* 1}$, C. J. Storm ${ }^{\dagger}$ \\ A. C. van Benthem, ${ }^{\dagger}$ and B. Buis ${ }^{\dagger}$ \\ *Department of Electrical Engineering, University of Twente, P.O. Box 217 \\ 7500 AE Enschede, The Netherlands Email: C.H.SLUMP.@EL.utwente.nl \\ ${ }^{\dagger}$ Cardiology Department. University Hospital Leiden, The Netherlands
}

(Received 17 June 1993; revised 12 August 1994)

\begin{abstract}
In this paper we report results from an ongoing study about the diagnostic benefits of three-dimensional (3D) visualization and quantification of stenosed coronary artery segments. Biplane angiographic images do not provide enough information for the exact reconstruction of the coronary arteries. Therefore, a priori information about the 3D shape to be reconstructed must be incorporated into the reconstruction algorithm. One approach is to assume a circular cross-section of the coronary artery. Hence, the diameter is estimated from the contours of the vessel in both projections. Another approach is based on densitometry and searches for a solution of the reconstruction problem close to the previously reconstructed adjacent slice. In this paper we apply contour information as well as the densitometrical profiles of the two orthogonal vessel projections. We present a new probabilistic densitometric reconstruction algorithm, which extends the correct handling of the stochastic properties of the density profiles into the network flow based reconstruction algorithm. The reconstructed coronary segment is visualized in three dimensions. In order to assess the accuracy of the reconstruction, the method is applied to tubes with artificial obstruction of known geometry, modeling coronary artery stenoses. These catheter tubes are filled with normal iodine contrast material. The results of the reconstruction and visualization are discussed with respect to clinical usefulness.
\end{abstract}

Key Words: 3D reconstruction, 3D display, Densitometry, Coronary arteriography, Biplane acquisition

\section{INTRODUCTION}

With coronary arteriography the assessment of the clinical relevance of a stenosis in a coronary artery is complicated and prone to error. Even in the case of biplane imaging where two orthogonal views of the stenosed vessel segment are acquired, the interpretation requires much skill. A good overview about the quantitative assessment of stenosed coronary arteries can be found in Reiber et al. (1).

This paper reports about a part of a larger study devoted to the question of whether quantification and three-dimensional (3D) visualization of a stenosed artery will benefit the cardiologist in the diagnostic task mentioned abovc. A previous paper (2) in this study was based upon the assumption that the cross-sections of the vessels are circular. In (2) the reconstruction and visualization of the vessel and the assessment of the flow impedance based on the reconstructed geometry is presented by applying the developed algo-

Presented in part at the Fourth International Symposium on Coronary Arteriography, Rotterdam. The Netherlands. June 23-25. 1991.

${ }^{1}$ To whom correspondence should be addressed. rithms to clinical coronary biplane exposures. An alternative approach to $3 \mathrm{D}$ reconstruction and visualization is the calculation of a functional measure of the clinical relevance of the pertinent stenosis [i.e., the so-called Coronary Flow Reserve (CFR), e.g., (3, 4)]. In (5) we report a less complicated and demanding procedure than CFR, in which we determine densitometrically the relative flow distribution between the two main branches i.e. the Left Arterial Descending (LAD) and the Circumflex Artery (RCX) of the left coronary artery. The comparison between basal and pharmacologically induced hyperemic flow provides useful functional information about the relevance of stenoses situated in the vascular tree.

If the 3D geometry of a stenosis is known, the resulting flow can be calculated in principle by means of a fluid dynamical computation. However, the computational complexity of this inverse problem is prohibitive. The vascular geometry together with the boundary conditions are not known, since only recordings of flow patterns of injected contrast material are available. Therefore, in (2) our approach has been modest and we have assessed the flow impedance on the basis of a cylindric geometrical model of the shape of the stenosed artery segment. 


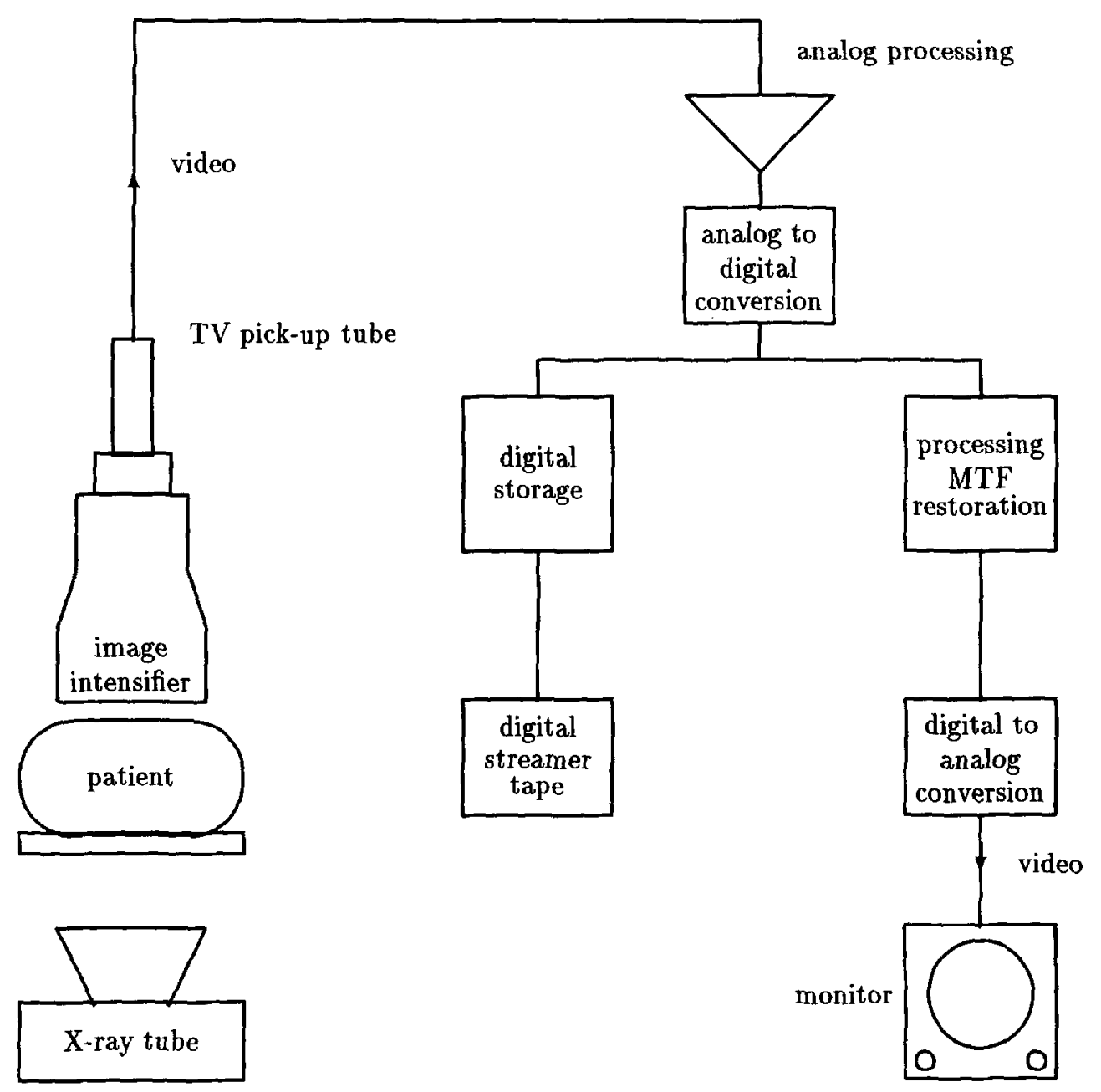

Fig. 1. Block diagram of Digital Cardiac Imaging (DCI), frontal acquisition.

In this paper we abandon the assumption of symmetric coronary vessels and allow asymmetric or even irregular cross sections, likely to occur in normal clinical practise. The main topic of this paper is the densitometric reconstruction of the stenosed artery segments from biplane projections. In (6) many of the older pertinent contributions to the literature are referenced, recent work on the 3D reconstruction of coronary arteries from biplane angiograms is [e.g. $(7,8$, $9)$ ). The approach in this paper is in line with previous work $(6,10,11)$ with digitized cine-film images. The ill-posed problem of binary 3D reconstruction from biplane exposures is regularized by incorporating into the reconstruction algorithm a priori information, such as the shape of the adjacent (previously reconstructed) slice.

In order to test the validity of the method, we reconstruct static phantom vessels with occlusions of known geometry. The artificial stenotic arteries consist of radiophaque catheter tubes filled with contrast ma- terial. Examples of the performance of the developed algorithms on the phantom data are shown with a clinical coronary artery as a final result. In the next section the image acquisition and data handling procedures are described. The reconstruction, display and phantom experiment are described in the subsequent sections.

\section{IMAGE ACQUISITION AND DATA HANDLING}

The images processed in the research reported in this paper, are acquired with a Philips Digital Cardiac Imaging (DCI) system which is the all digital cath. lab. The system is schematically depicted in Fig. 1 (only the frontal image acquisition is indicated). X-ray pulses are generated (typ. 5-8 ms) at a rate of 15-30 frames/ $s$ in order to freeze the motion of the coronary arteries which are injected with a contrast agent by means of a stable positioned coronary catheter. The $\mathrm{X}$-ray pho- 
tons which have traversed the patient are converted into a video image by the image intensifier-TV system. The resulting video signal is digitized in 8 bits after analog processing. The images are stored on a real time digital disk in $512 \times 512 \times 8$ bit up to $60 \mathrm{frames} / \mathrm{s}$ or in case of biplane up to $2 \times 30 \mathrm{frames} / \mathrm{s}$. Parallel to the storage on disk, the images are processed (i.e., the Modulation Transfer Function (MTF) is corrected by unsharp masking, see e.g. (12) in order to compensate for the lowpass filtering by the imaging components ) and displayed on a monitor. A versatile user-interface is provided for viewing and post-processing.

For our purpose selected clinical and phantom biplane runs from Leiden University Hospital were transferred from the disk and archived on a digital streamer tape in an off-line process. The tapes were transferred to the HP-Apollo network of workstations at the University of Twente for further off-line processing.

Specially prepared segments of catheters in the range of 4-12 french are filled with contrast material and positioned with respect to the frontal and lateral image intensifier. The catheter tubes have been prepared with a variety of artificial stenosis, that is symmetrical and asymmetrical, (relative) short and long narrowings, entrance resp. exit angle variations, etc. Obtaining a homogeneous catheter filling without air bubbles appeared to be not so simple and a special filling technique had to be developed. We have used a needle positioned close to the artificial obstruction as a reference point in both projections. The tubes have been imaged at normal magnification and X-ray parameter settings, additional perspex plates were placed at the X-ray collimator.

In general, the densitometric analysis of angiograms requires corrections to be applied to the images. A not exhaustive list includes correction of the nonlinear analog preprocessing (white compression), removal of background structures by log-subtraction of an empty mask image acquired prior to iodine injection (a cumbersome procedure in cardiac imaging due to object movements, however, very convenient in our case of imaging static phantom tubes), correction for pincushion distortion due to the curved detector screen of the image intensifier, restoration of the effects of Xray scatter and veiling glare. Also the differences between the frontal and lateral imaging chain should be taken into account. In our case we have positioned the phantom tubes such that the stenosed part is projected near the resp. center of the image intensifier and with the frontal and lateral geometrical magnifications approximately equal. Therefore, we discard corrections for pincushion distortion and magnification differences.
In general the data handling and reconstruction procedure contains the following steps:

1. Selection of the pair of orthogonal images showing the stenosed vessel part.

2. Selection of the region of interest, identification of reference points such as, for example, the catheter tip.

3. Preprocessing, lateral shift for aligning the projections (2), (optional) subtraction of a prerecorded empty mask (13). As the X-ray control unit sets the $\mathrm{X}$-ray parameters $\mathrm{kVp}$ and $\mathrm{mA}$ such that a fixed average video level is attained, this subtraction is a first order (linear) correction for the effects of scatter, glare, vignetling and white compression.

4. In the selected image regions the contours of the stenosed vessel segments are traced manually under cursor control (2), the selected contour points are stored in a file together with the extracted density profiles.

5. Reconstruction slice by slice using the probabilistic minimum cost algorithm. The next section describes this procedure in greater detail.

6. Entering viewing angles and 3D surface rendering with shading followed by 3D display of the stack of slices.

\section{DENSITOMETRIC 3D RECONSTRUCTION}

In this section we describe the probabilistic densitometric reconstruction algorithm for coronary artery segments from biplane projections. Orthogonal biplane images which are (almost) simultaneously acquired, are required for the reconstruction. In both projections the contours of the vessel are traced, as described in the previous section. The axis of the vessel segment should not be parallel to one of the X-ray beams. In practice, the angle must be more than 10 degrees with the $x z$-plane ( see the projection geometry as indicated in Fig. 2). Note the left orientation of the coordinate frame, which is often used in 3D computer graphics.

We assume that the vessel segment of interest is homogeneously filled with contrast agent, and that the $\mathrm{X}$-ray absorption is mainly due to the contrast agent. The latter condition is certainly fulfilled for the case of our prepared tubes. At this stage we neglect X-ray quantum noise, scattered radiation and beam hardening. The reconstruction problem can be described as a binary detection task in which we have to detect the presence absence of injected X-ray contrast agent in each volume ccll in 3D. Further simplifications of the acquisition model, such as assuming that the $\mathrm{X}$ ray beams can be considercd to be parallel over a length of the order of the size of the cross section of the vessel 
frontal image detector

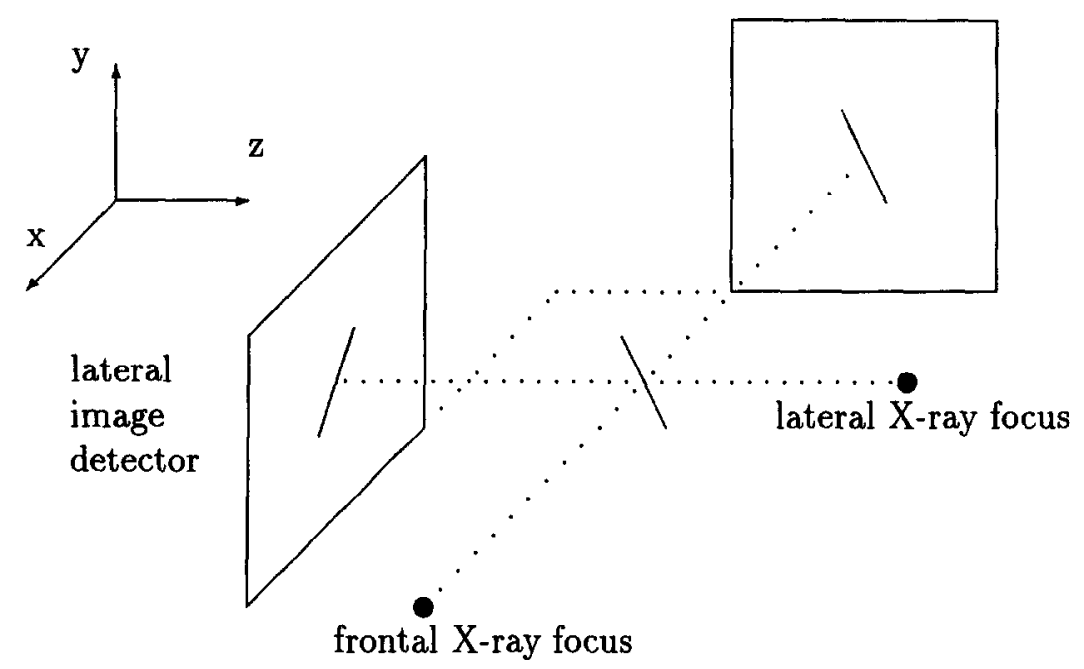

Fig. 2. The projection geometry and the coordinate frame.

and dividing the vessel segment into a stack of parallel slices, reduce the complexity of the reconstruction problem (6). Each 2D slice has to be reconstructed from its two one-dimensional absorption profiles which correspond with the row resp. column sums of a binary matrix. This image matrix represents the cross section of the vessel. The entries equal to one denote the presence of contrast agent in the cross section, a zero indicates the absence of contrast.

Let $X$ be a $m \times n$ matrix with entries $x_{i, j} \in\{0$, $1\}$, where $\alpha_{i}$ represents the row sums of $X$ and $\beta_{j}$ represents the column sums.

The reconstruction problem is to determine the $x_{i, j}$ subject to:

$$
\begin{aligned}
& \sum_{j=1}^{n} x_{i, j}=\alpha_{i}, i=1, \ldots, m, \\
& \sum_{i=1}^{m} x_{i, j}=\beta_{j}, j=1, \ldots, n,
\end{aligned}
$$

with the condition that total mass density $S$ of the pertinent slice is equal to the total row sum and is equal to the total column sum:

$$
S=\sum_{i=1}^{m} \alpha_{i}=\sum_{j=1}^{n} \beta_{j}
$$

In general this problem is underdetermined and has many solutions. To regularize this ill-posed problem, additional, for example a priori information is necessary to obtain a unique solution. We base our approach (6) on the shape similarity between consecutive slices. The vessel segment is reconstructed sliceby-slice and we recursively use information from the adjacent, previously reconstructed slice. We therefore definc an $m \times n$ matrix $C$ of cost coefficients with entries $c_{i, j}$ which denote the cost of assigning $x_{i, j}$ equal to one. The $c_{i, j}$ coefficients are proportional to the distance to the contour of the adjacent slice.

We now have the following optimization problem:

$$
\text { Minimize } \sum_{i} \sum_{j} c_{i, j} x_{i, j}
$$

subject to the constraints (Eqs. 1-3). It is convenient to consider this integer optimization problem as a directed capacitated network flow. A capacitated directed network consists of nodes and directed arcs connecting the nodes. We have two special nodes, the source and the sink, and two sets of intermediate nodes. The source is connected with the $m$ nodes of the first set of intermediate nodes. Likewise, the sink node is connected to the $n$ nodes of the second set. Directed arcs connect each node of the first set with each node of the second set. A directed arc is able to transport in its direction a discrete number of flow units less than or equal to its capacity. The capacity of the intermediate arcs is equal to one. If we set the capacity of the source and sink arcs equal to the mcasured row and column sums, $\alpha_{i}, i=1, \ldots, m$ and $\beta_{j}, j=1, \ldots, n$, respectively, we obtain a maximal flow of $S$ units of flow through the network (6). As indicated in Fig. 3 the flow through the intermediate arcs corresponds with the binary matrix $X$ satisfying (Eqs. 1-3). By assigning the cost coefficients $c_{i, j}$ to the pertinent intermediate arcs, the cost minimization problem (4) is stated as the network flow problem of sending $S$ units of flow from source to sink at the lowest costs given the capacities of the source 


\begin{tabular}{|c|c|c|c|c|}
\hline 0 & 1 & 1 & 0 & 0 \\
\hline 0 & 1 & 1 & 1 & 0 \\
\hline 1 & 1 & 1 & 1 & 1 \\
\hline 0 & 1 & 0 & 1 & 0 \\
\hline
\end{tabular}

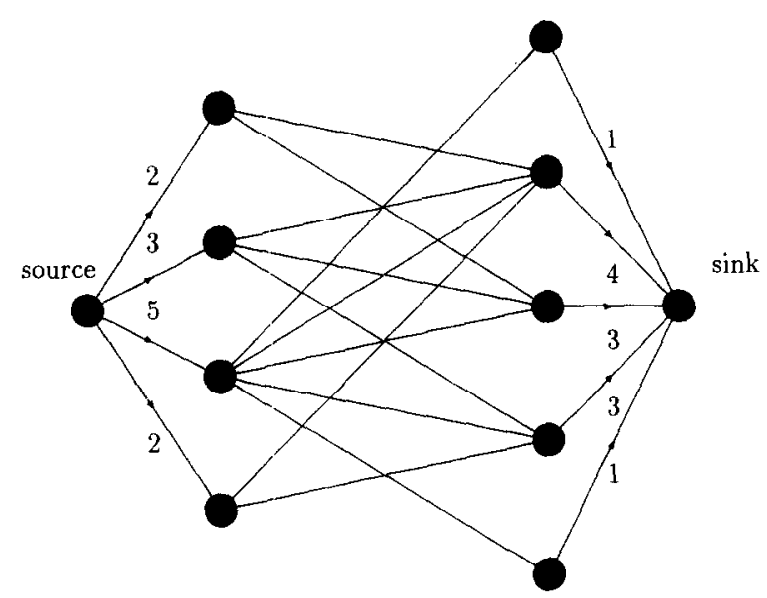

Fig. 3. An example showing the equivalence of the binary reconstruction problem from row and column sums with the flow in a capacitated directed network. The capacities of the source and sink arcs are equal to the row and column sums, respectively. The drawn intermediate arcs do transport a unit of flow equivalent with a corresponding entry in the matrix equal to unity.

and sink arcs. The advantage of this approach (6) is that efficient algorithms from the field of operations research can be applied, for example, the maximal flow algorithm (14) and the minimum cost algorithm (15).

So far we have neglected the $X$-ray quantum noise. Because of the high frame rate (typ. 30-60/s) at which the images are acquired in cardiac catheterization, the number of $\mathrm{X}$-ray quanta per frame is limited. X-ray quantum noise is present in the acquired images and the measured density profiles have become realizations of a stochastic process. In (11) we have analyzed the effects of quantum noise on the measured density profile, neglecting scatter and assuming an ideal X-ray detector i.e. with MTF equal to unity for all pertinent spatial frequencies. Due to the noise it is highly unlikely that there exists a solution to (Eqs. 1-3). We transform the reconstruction problem in a probabilistic minimum cost flow in a capacitated directed network. The first step is to estimate the number of flow units $\hat{S}$ to be transported from source to sink in the network (we use the " $\wedge$ " symbol to denote stochastic variables). $\hat{S}$ is proportional to the projected total mass density in the pertinent slice, it is estimated by the average of the total stochastic row sum and the total stochastic column sum:

$$
\hat{S}=\frac{1}{2}\left(\sum_{i=1}^{m} \hat{\alpha}_{i}+\sum_{j=1}^{n} \hat{\beta}_{j}\right)
$$

To the source and sink arcs a cost function is assigned proportional to the probability distribution of the pertinent row and column sums. The solution to the reconstruction problem is now obtained by sending the $\hat{S}$ flow units from source to sink at the lowest cost. In (11) results of this approach are shown. In (16) the $\mathrm{X}$-ray quantum noise is characterized in a more realistic way, incorporating the spatial filtering by the nonideal image intensifier-television system. The modeling of noise has been the subject of many papers, see the references cited in (16). We base our characterization of the noise in the density profiles on (16), neglecting the electrical noise of the imaging system components. If there is no object in the X-ray beam, the random number $\hat{n}$ of photons impinging on the image intensifier input screen per $\mathrm{mm}^{2}$ is Poisson distributed:

$$
P(\hat{n}=k)=e^{-\lambda} \frac{\lambda^{k}}{k !}, \quad k=0,1,2, \ldots
$$

with $\lambda$ the average number of photons per $\mathrm{mm}^{2}$. At the output screcn of the image intensifier the incoming photons are imaged with the two-dimensional shot noise process $i_{o}\left(x_{o}, y_{o}\right)$,

$$
i_{v}\left(x_{o}, y_{o}\right)=\eta c \sum_{i} h\left(x_{o}-x_{i}, y_{v}-y_{i}\right)
$$

with $\left(x_{i}, y_{i}\right)$ the random positions of the detected incoming photons, $h($, ) the two-dimensional spatial impuls response characterizing the imaging system, $\eta$ the Detection Quantum Efficiency (DQE) of the detection process and the total detection gain is denoted by $c$. According to Campbell's theorem $(17$, p. 561$)$ the expectation value of the output stochastic process is given by:

$$
E\left(i_{o}\left(x_{o}, y_{o}\right)\right)=\lambda \eta c \int_{-\infty}^{\infty} \int_{-\infty}^{\infty} h(x, y) d x d y,
$$

and the variance is given by:

$$
\sigma^{2}\left(i_{o}\left(x_{o}, y_{o}\right)\right)=\lambda \eta c^{2} \int_{-\infty}^{\infty} \int_{-\infty}^{\infty} h^{2}(x, y) d x d y .
$$


We can now characterize the stochastic image process by the signal-to-noise ratio:

$$
\frac{S}{N}=\sqrt{\frac{\lambda \eta}{\int_{-\infty}^{\infty} \int_{-\infty}^{\infty} h^{2}\left(x_{o}, y_{o}\right) d x_{o} d y_{o}}}
$$

or $(18,19)$ expressed in the system's MTF:

$$
\frac{S}{N}=\sqrt{\frac{\lambda \eta}{2 \pi \int_{0}^{\infty} v M T F^{2}(v) d v}}
$$

The influence of the lowpass spatial filtering as expressed in the systems MTF is an improvement in the signal-to-noise ratio. With an ideal detector characterized by $M T F=1$ we obtain the SNR:

$$
\frac{S}{N}=\sqrt{\lambda \eta A_{c}}
$$

with $A_{\mathfrak{e}}$ the area of a pixel. For non-ideal MTF's $A_{e}$ represents the effective noise sampling aperture (18, 19):

$$
A_{e}=\left(2 \pi \int_{0}^{\infty} v M T F^{2}(v) d v\right)^{-1} .
$$

For a typical image intensifier-television chain in the 9 " mode with $8 \mu R /$ frame, the theoretical signalto-noise ratio is $\approx 16$. In close agreement with the MTF of the system, we have measured with acquired digital images a $\frac{S}{N} \approx 50(16)$.

In order to obtain the row and column sums from the pertinent frontal, respectively lateral projection image wc proceed according to standard practice in Digital Subtraction Angiography (DSA). For reasons of noise reduction, we gencrate a so-called empty mask image by averaging a large number of exposures before the iodine signal is present in the image. The logarithm of the exposure containing the iodine signal is subtracted from the logarithm of the empty mask image. In good approximation for narrow X-ray beams and with neglect of scattered radiation the empty mask image is proportional to:

$$
\lambda_{m}=\lambda_{o} e^{-\mu_{m} d_{m}}
$$

with $\lambda_{m}$ the average number of (detected) X-ray quanta behind the imaged object, $\lambda_{o}$ the average number of quanta in the exposure, $\mu_{m}$ the total attenuation coefficient $\left(\mathrm{cm}^{-1}\right)$ of the imaged object and $d_{m}$ the pertinent effective thickness $(\mathrm{cm})$. In order to simplify the notation, the dependence of all quantities on the spatial coordinates has been dropped. The image containing the iodine signal is a realization of a stochastic process, with expectation:

$$
E\left(i_{o, \text { iodine }}\right)=\lambda_{\text {iodine }}=\lambda_{o} e^{-\mu_{m} d_{m}-\mu_{\text {iodinediodine }}} .
$$

The effective iodine thickness $d_{\text {iodine }}$ equals the row $\alpha_{i}$ respectively column $\beta_{j}$ sum value to be determined times the pixel size $d_{p i x e t}$ :

$$
\left.\begin{array}{c}
\alpha_{i} \\
\beta_{j}
\end{array}\right\}=\frac{d_{\text {iodine }}}{\mu_{\text {iodine }} \times d_{\text {pixel }}} .
$$

The DSA subtraction results in:

$$
\left.\begin{array}{c}
\hat{\alpha}_{i} \\
\hat{\beta}_{j}
\end{array}\right\}=\frac{\log \left(i_{o, \text { mask }}\right)-\log \left(\hat{l}_{o, \text { iodine }}\right)}{\mu_{\text {iodine }} \times d_{\text {pixel }}} .
$$

Because the log function is a smooth function and the pertinent probability distribution of the projection data is peaked around the expectation value we obtain in good approximation that the expectation value of the row respectively column sum is equal to the true value (i.e., the estimates are unbiased). For the variance we obtain in the same way $(17$, p. $151-152)$ :

$$
\sigma_{\alpha_{i}, \beta_{j}}^{2}=\left(\left(\mu_{\text {iodine }} d_{\text {pixel }}\right)^{2} e^{-\mu_{\text {iodine }} d_{\text {iodine }}}\right)^{-1} \sigma^{2},
$$

with $\sigma^{2}$ given in Eq. (9). From this result we see that higher row and column sums do have a larger variance, which is intuitive pleasing because in this case less quanta are detected.

We now assign a parabolic cost function to the source and sink arcs in the network flow description (11). The width of the cost function is chosen proportional to $\sigma_{\alpha_{i} \beta_{j}}^{2}$ because it reflects the uncertainty in the measurement of the projection values. A small variance corresponds with less uncertainty and thus a steep (parabolic) cost function centered around the obtained values for $\hat{\alpha}_{i}, \hat{\beta}_{j}$. A large variance reveals more uncertainty which is reflected in a larger width of the cost function. The total procedure is clarified by the following example. Suppose we image a 10 french catheter on the $16 \mathrm{~cm}$ II format (6.5") at a geometrical magnification of 1.6 , we have for the $d_{\text {pixel }}$ of $0.31 \mathrm{~mm}$ an iodine shadow projection of $5.3 \mathrm{~mm}$ in diameter, corresponding to about 17 pixels. At $75 k V_{p}$ the effective mass attenuation coefficient of iodine $(\mu / \rho)_{\text {iodine }}$ equals $(21$, p. 87$) 13 \mathrm{~cm}^{2} / \mathrm{g}$. With an iodine mass density $\rho$ of $270 \mathrm{mg} / \mathrm{cm}^{3}$ the iodine filled catheter reduces at its center the photon fluence by $69 \%$. The product of $\mu_{\text {iodine }} \times d_{\text {pixel }}$ equals 0.11 . For the case of the image intensifier-TV subsystem (16) in the 6.5" mode, the effective noise sampling aperture $A_{e}$ equals $1.12 \mathrm{~mm}^{2}$. Imaging with $75 k V_{p}$ and $1.5 \mathrm{~mm} \mathrm{Cu}$ prefilter at the $\mathrm{X}$ ray collimator we have $60 \mathrm{keV}$ effective, corresponding with $3.2 \times 10^{14}$ quanta $/ m^{2}$ per $R$. With an exposure of 
$16 \mu R /$ frame exposure at the input of the II and a $D Q E$ $\eta$ of $60 \%$, we have according to Fq. (9) and Eq. (12) a $\sigma^{2}$ equal to:

$$
\sigma^{2}=\frac{S_{i g n a l}}{\lambda \eta A_{e}}=4.76
$$

for a signal level $S_{\text {ignal }}$ at half the AD converter range of 8 bits. With the above numbers, the $\sigma_{\alpha_{y}, \beta_{j}}^{2}$ of the estimated projections values $\hat{\alpha}_{i}, \hat{\beta}_{j}$ and thus the width of the cost function in the probabilistic network flow reconstruction algorithm is fully specified.

\section{RESULTS}

In this section the probabilistic densitometric reconstruction algorithm described in the previous section, is applied to two phantom tube segments with known occlusion. In addition we present one clinical case. Of each stenosed segment, the acquired frontal and lateral X-ray projections are presented, followed by 3D visualization of the pertinent segment for two different viewing angles. For the display of the stack of reconstructed slices the coordinates of the surface points are required. Similar to (2) for circular cross sections we represent the contour of the reconstructed slice by a polygon. The surface of the reconstructed segment is build by triangles between adjacent polygon-shaped slices. For circular cross sections the tiling of the surface is quite simple if each polygon has the same number of vertices.

The densitometric reconstructions are more irregular and therefore it is not possible to define the shape of the cross sections with a fixed number of vertices at known angles. The adjacent slices represented by polygons have to be connected by triangles. For this

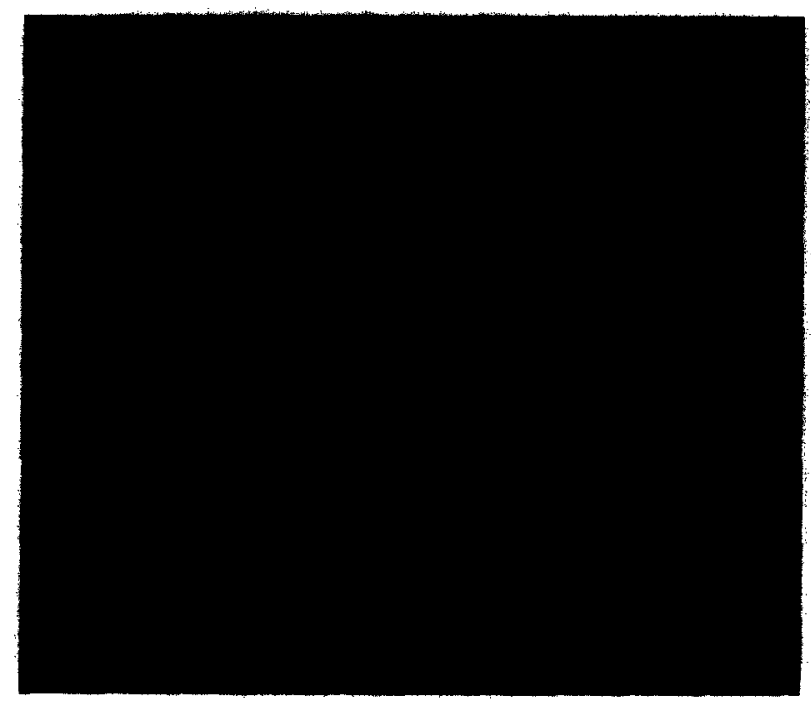

Fig. 4. Frontal angiogram first example.

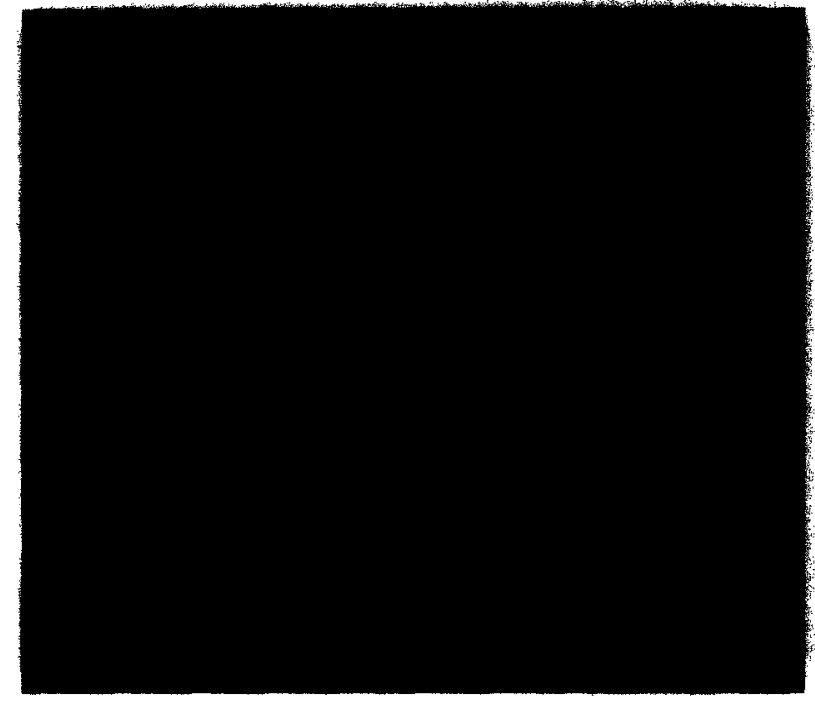

Fig. 5. Lateral angiogram first example.

purpose a special tiling procedure was developed. The search procedure starts generating triangles for a pair of corresponding vertices on the two pertinent polygons. The algorithm stops when the starting vertices are reached again. We apply the software package 3D GMR (20) for the display of the stack of reconstructed slices. In order to apply this package we need to specify the position of a light source and a viewing angle in the $x y z$-frame as well. The reflected light intensities from the triangular surfaces are calculated using interpolation according to the Phong shading algorithm (22) and displayed as an image.

The frontal and lateral projection of the first example, a 12 french catheter tube are presented in Figures 4 and 5, respectively. Note the presence of a needle which is visible in both projections and is used as a reference marker to relate the two projections with respect to each other. The reconstructed results for different viewing angles are shown in Figures 6 and 7. The next phantom example is a 12 french catheter with an asymmetric narrowing, Figures 8 and 9 do contain the projections and Figures 10 and 11 the reconstructed results. In the final example we present a clinical case. Fig. 12 and 13 do contain the frontal and lateral projection of a stenosed right-coronary artery. The result of the reconstruction of the stenosed segment is shown for several viewing angles in Fig. 14-16.

\section{DISCUSSION OF THE RESULTS}

In comparison with previous results (2) based on circular cross sections, the vessel segments reconstructed by the probabilistic densitometric reconstruction algorithm do appear to have rather rough walls. 


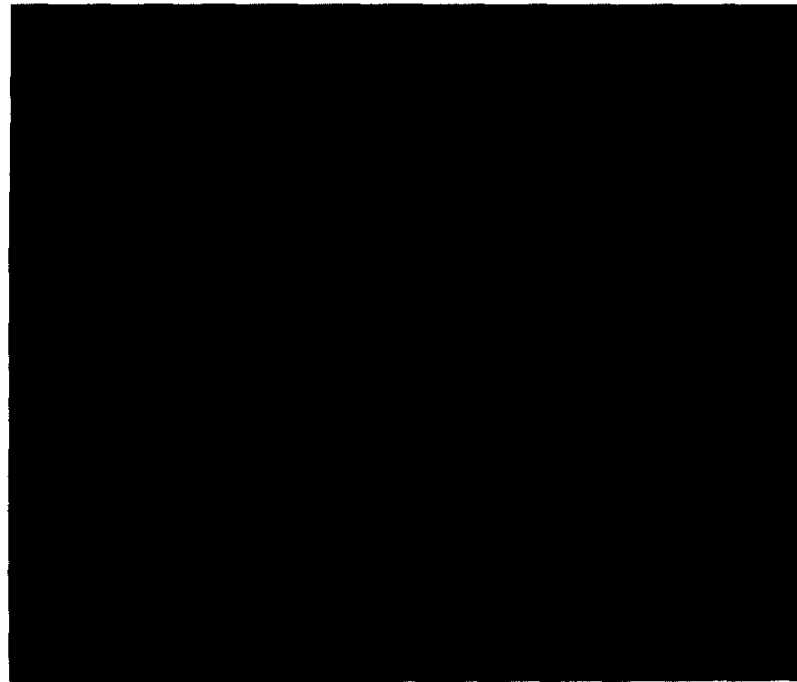

Fig. 6. Reconstructed view of the stenosed arterial segment of Figs. 6 and 7.

Our first impression was a little bit of a disappointment. On the other hand the asymmetric occlusion does show up in the reconstruction result, not visible for the circular cross section method. Second thoughts about the roughness leads us to the following analysis. Due to noise variations the stack of slices even of a cylindrical tube does show up irregular. At no occasion in the set of phantom tubes, the reconstructed result diverged from the true shape. The tiling procedure which is a crucial non-trivial step for finding a good match for the triangles, tends to enhance the roughness. Finally, the Phong shading algorithm (22) we apply is based

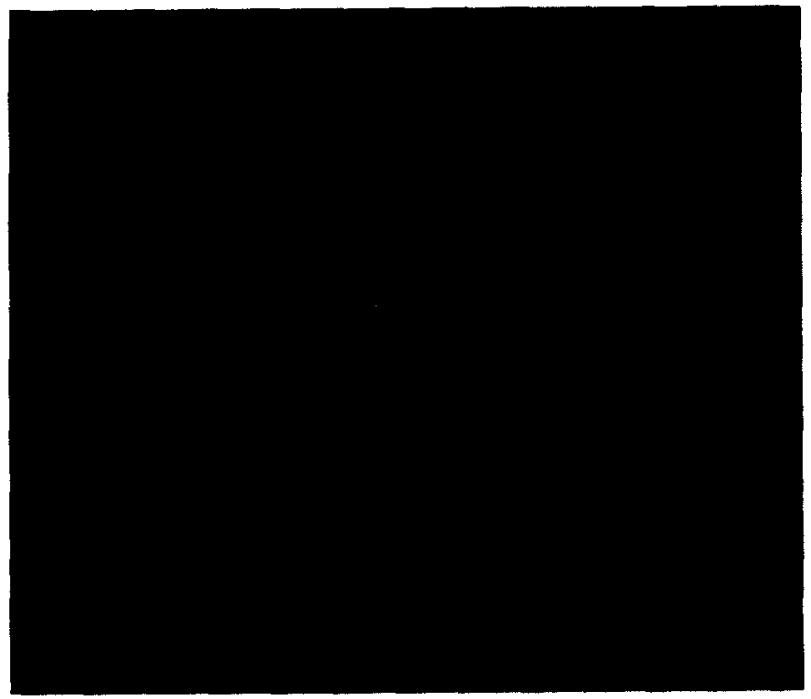

Fig. 7. Reconstructed view of the stenosed arterial segment of Figs. 6 and 7.

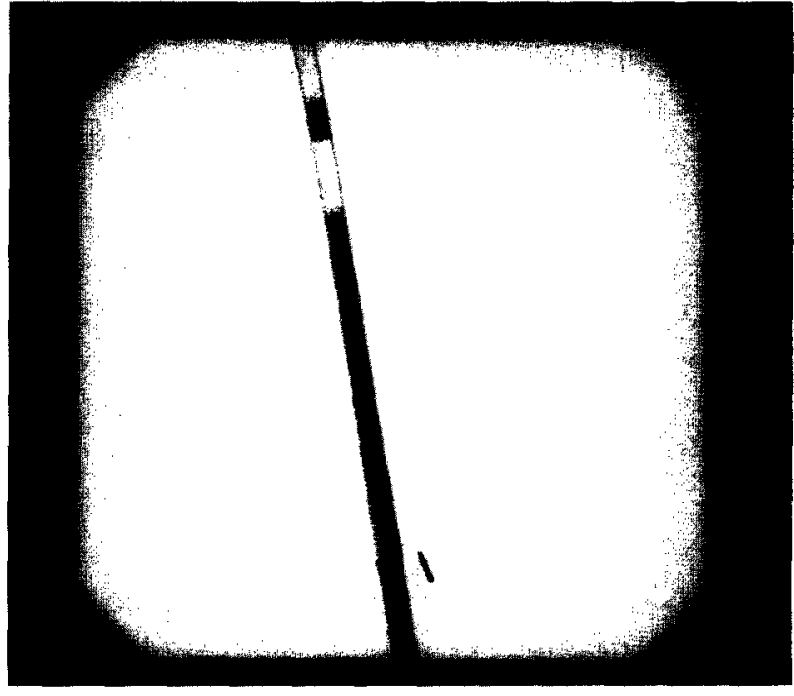

Fig. 8. Frontal angiogram third example.

upon second order interpolation and requires the calculation of spatial derivatives. Existing small discontinuities are greatly enhanced by this procedure. Also the position of the light source is important. Viewing a surface with light coming in with a large angle with respect to the normal on that surface, enhances the conspicuity of small surface variations. The reconstruction algorithm is based upon a slice-by-slice approach. In order to increase the local continuity of the reconstructed vessel we will continue our research for reconstruction algorithms treating the stenosed vessel segments as a whole. One of our goals is the calculation of the flow impedance based on the reconstructed ge-

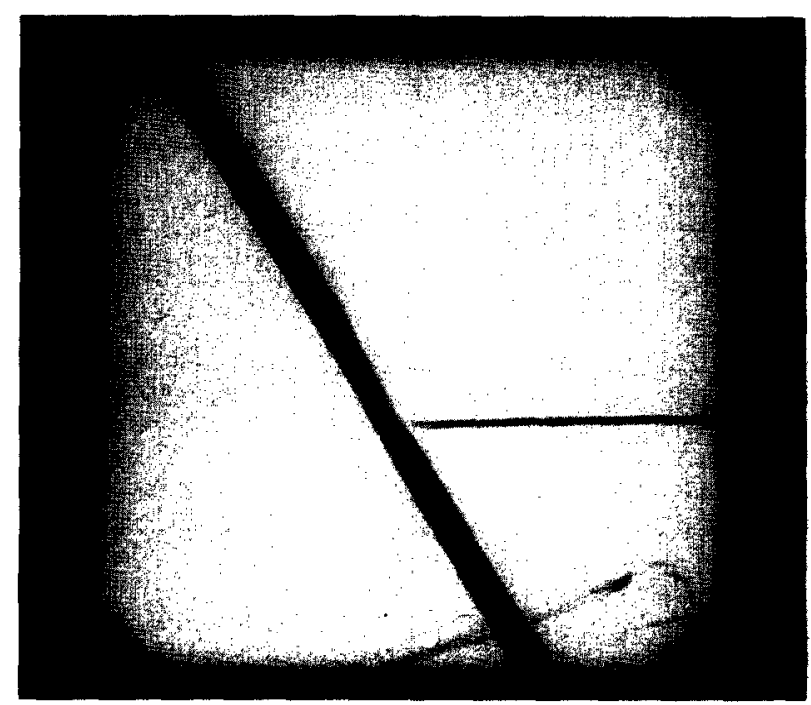

Fig. 9. Lateral angiogram third example. 


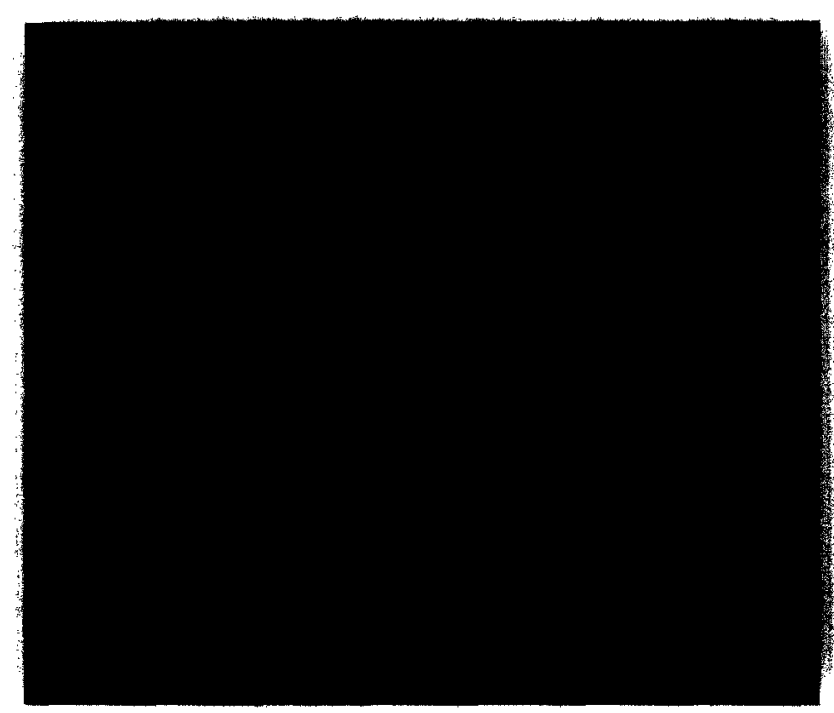

Fig. 10. Reconstructed view of the stenosed arterial segment of Figs. 8 and 9.

ometry. The present results are not suitable for this purpose. However, we expect that $3 \mathrm{D}$ visualization and quantification of the flow impedance of the stenosis are useful diagnostic tools, provided the computations can be carried out on-line between cardiac exposure runs. The realization of this goal is still far away and will require much effort.

\section{SUMMARY}

This paper is a part of an ongoing study about the diagnostic benefits of 3D visualization and quantif-

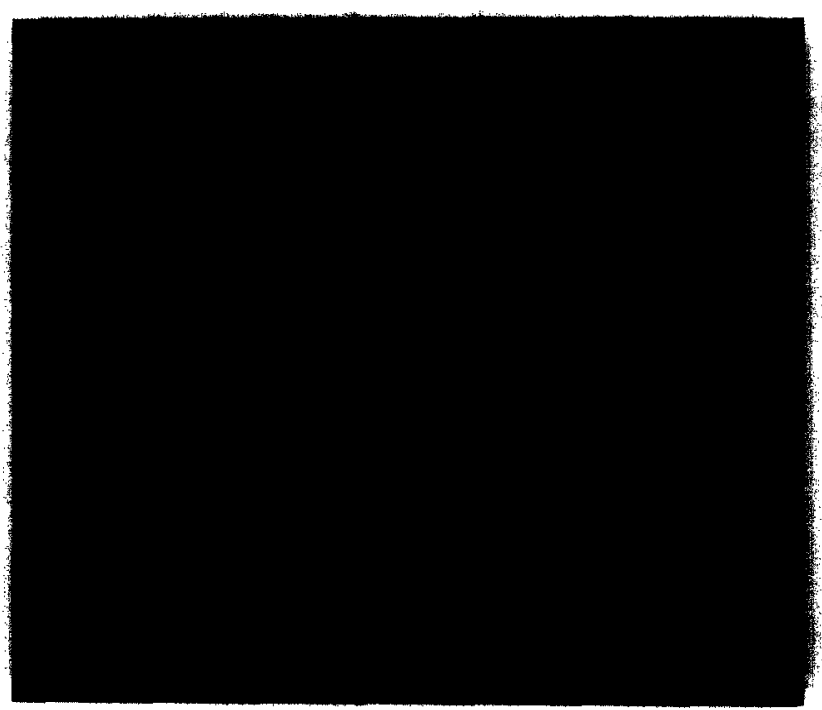

Fig. 11. Rcconstructed view of the stenosed arterial segment of Figs. 8 and 9.

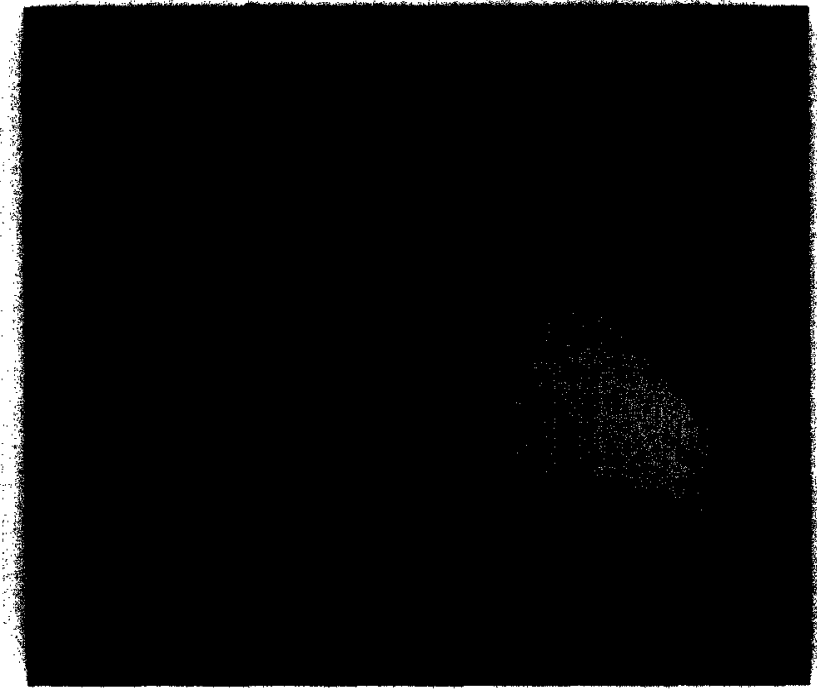

Fig. 12. Frontal angiogram clinical example.

cation of stenosed coronary artery segments. Biplane angiographic images do not provide enough information for the reconstruction of the coronary arteries. Therefore, a priori information about the $3 \mathrm{D}$ shape to be reconstructed must be incorporated into the reconstruction algorithm. One approach is to assume a circular cross-section of the coronary artery. Hence, the diameter is estimated from the contours of the vessels in both projections.

In this paper we base the reconstruction on densitometric information slice-by-slice. We search for a solution of the reconstruction problem close to the previously reconstructed adjacent slice. We apply con-

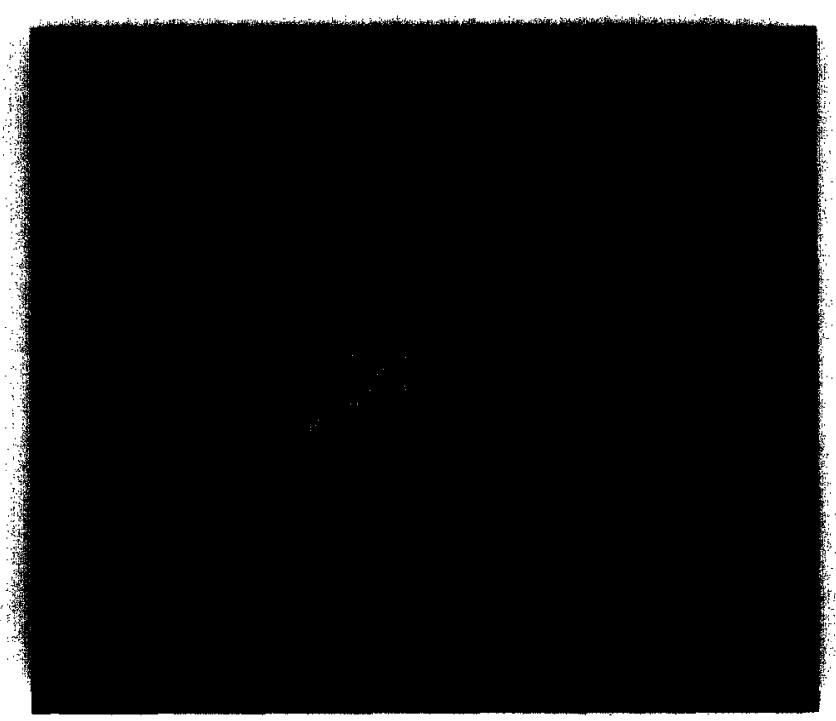

Fig. 13. Lateral angiogram clinical example. 


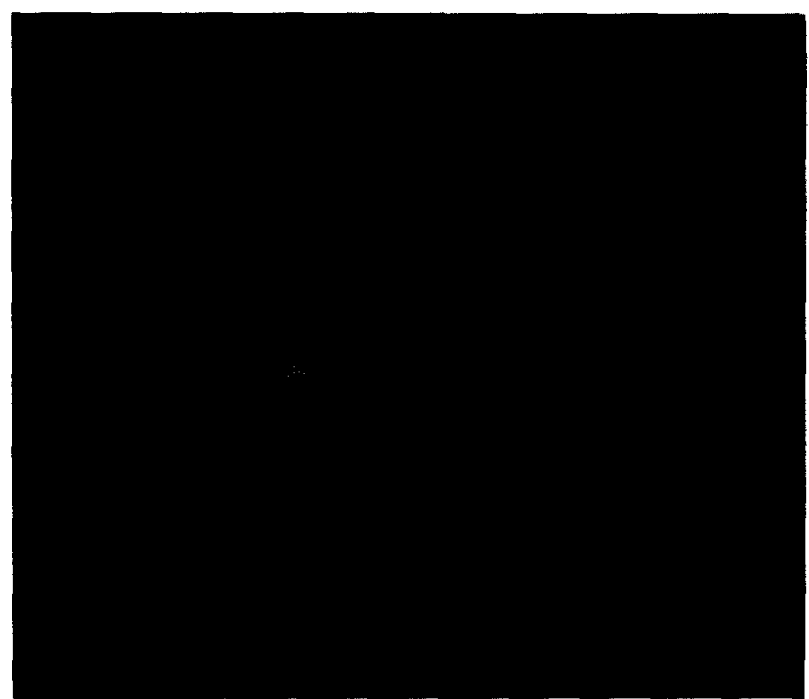

Fig. 14. Reconstructed view of the stenosed arterial segment of Figs. 12 and 13.

tour information as well as the densitometrical profiles of the two orthogonal vessel projections which are transformed to the row and column sums, respectively, of a binary matrix.

We present a new probabilistic densitometric reconstruction algorithm, which extends the correct handling of the stochastic properties of the density profiles into the network flow based reconstruction algorithm. The equivalence of the binary reconstruction problem from row and column sums with flow though a capacitated network has been shown by us before.

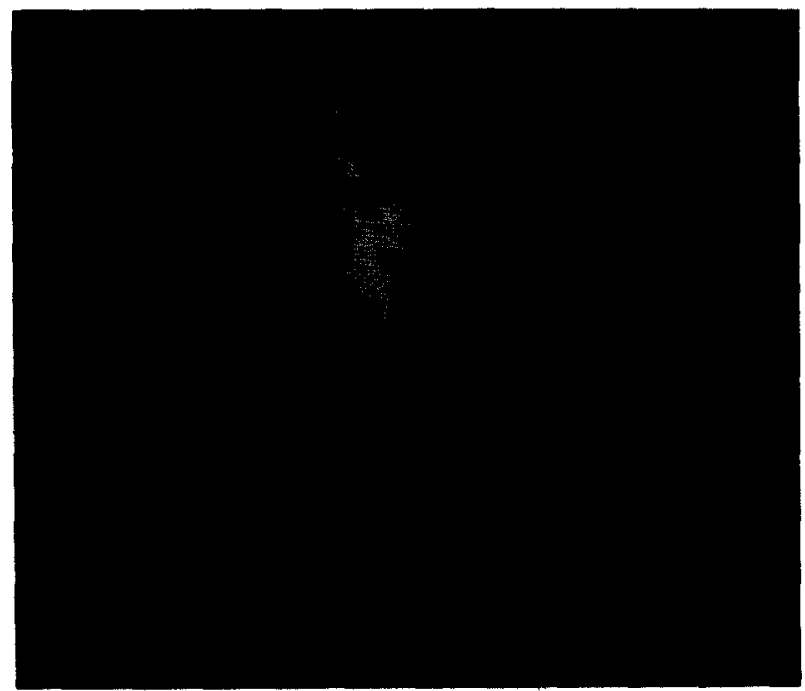

Fig. 15. Reconstructed view of the stenosed arterial scgment of Figs. 12 and 13.

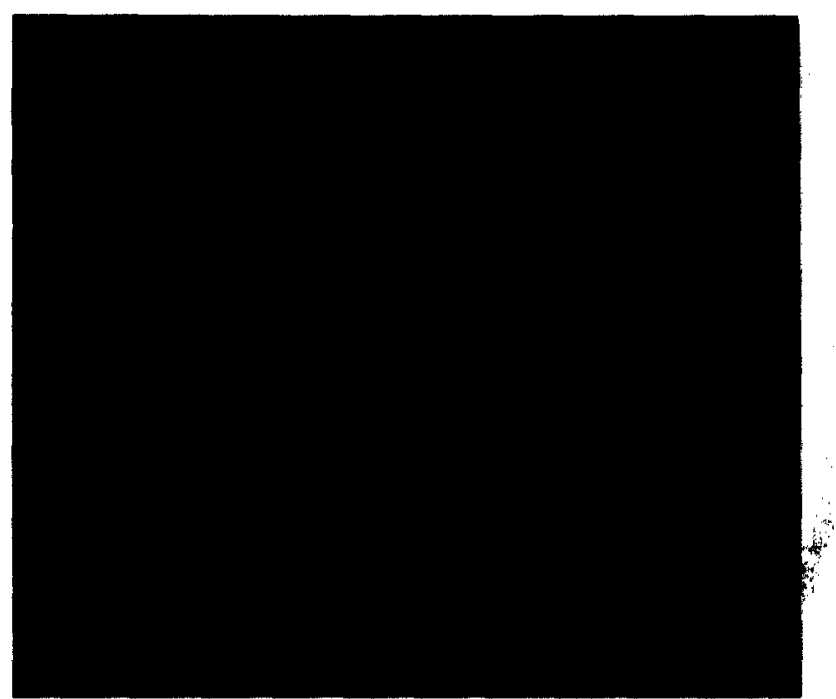

Fig. 16. Reconstructed view of the stenosed arterial segment of Figs. 12 and 13.

The cost function associated with a flow realization through the network is used to model a priori information about the solution to be obtained.

New to this approach is the assigning of a cost function to the source and sink arcs of the network, such that the width of the convex costfunction is inverse proportional to the variance of the probability distribution of the noisy densitometric projection data (i.e., the row and column sums, respectively).

The reconstructed coronary segment is visualized in three dimensions. In order to assess the accuracy, the method is applied to tubes with artificial obstruction of known geometry, modeling coronary stenoses. These catheter tubes are filled with contrast material. Two typical cases are shown together with one clinical example. The results of the reconstruction and visualization are discussed with respect to clinical usefulness. The results do appear to have rather rough walls which turns these results not suitable for flow impedance calculations. On the other hand, asymmetric occlusions not visible with circular based reconstruction methods, do show up clearly. We will continue this research with the development of densitometrical reconstruction algorithms not based on a slice-by-slice approach in order to preserve the local continuity.

Acknowledgments-The help of Mr. H. Stegehuis M.Sc. from Philips Medical Systems, Best, the Netherlands, in making the hardcopies of the images presented in this paper is gratefully acknowledged.

\section{REFERENCES}

1. Reiber, J.H.C.; Serruys, P.W.; Slager, C.J. Quantitative coronary and left ventricular cineangiography. Dordrecht, The Netherlands: Martinus Nijhoff Publishers; 1986. 
2. Hulzebosch. A.A.; Slump, C.H.; Viergever, M.A. Three-dimensional reconstruction of stenosed coronary artery segments with assessment of the flow impedance. Int. J. Cardiac Imaging 5: $135-143 ; 1990$

3. Vogel, R.; LeFree. M.; Bares, E.; O'Neill, W.; Forster. R.; Kirlin, P.: Smith, D.: Pitt, B. Application of digital techniques to selective coronary arteriography: Use, myocardial contrast appearance time to measure coronary flow reserve. Am. Heart J. 107:153$164 ; 1884$.

4. Cusma, J.T.; Toggart, E.J.; Folts. J.D.; Peppler, W.W.; Hangiandreou. N.J.: Lee, C.-S.; Mistretta, C.A. Digital subtraction angiographic imaging of coronary flow reserve. Circulation. 75 : 461-472; 1987

5. Lubbers, A.P.G.; Slump. C.H.; Storm, C.J. Digital densitometric determination of reative coronary flow distributions. In: Acharya, R.S., Goldgof, D.B., eds. Biomedical image processing and biomedical visualization. SPIE 1905, to appear, IS\&T/SPIE 1993 International Symposium on Electronic Imaging: Science \& Technology, San Jose, CA; 1993.

6. Slump, C.H.; Gertirands. J.J. A network flow approach to reconstruction of the left ventricle from two projections. Comput. Graph. Image Process. 18:18-36; 1982.

7. Garreau, M.: Coatrieux, J.L.: Collorec, R.; Chardenon, C. A knowledge-based approach for 3-D reconstruction and labcling of vascular networks from biplane angiographic projections. IEEE Trans. Med. Imag. 10:122-131; 1991.

8. Van Tran, L.; Bahn, R.C.; Sklansky, J. Reconstructing the cross sections of coronary arteries from biplane angiograms. IEEE Trans. Med. Imag. 11:517-529; 1992.

9. Pellot, C.; Mermert, A.: Sigelle, M.; Horain, P.; Maitre, M.: Peronneau, P. A 3L) reconstruction of vascular structures from two $\mathrm{X}$-ray angiograms using an adapted simulated annealing algorithm. IEEE Trans. Med. Imag. 13:48-60; 1994.

10. Reiber, J.H.C.; Gerbrands, J.J.; Troost, G.J.; Kooijman, C.J.; Slump, C.H. 3D reconstruction of coronary arterial segments from two projections. In: Heintzen, P.H.; Brennecke, R., eds. Digital imaging in cardiovascular radiology. Stuttgart: Thieme: 1983:151-163.

11. Gerbrands. J.J.: Slump, C.H. 3D reconstruction of homogeneous objects from two Poisson-distributed projections. Pattern Recognition Letters $3: 137-145 ; 1985$.

12. Gonzalez. R.C.; Wouds. R.E. Digital image processing. Reading. MA: Addison-Wesley, 1992.

13. Cowen, A.R.; Haywood, J.M.; Clarke, O.F.; Rause, S. Digital grey-scale fluography: a new approach to radiographic imaging. Br. J. Radiol. 57:533-538; 1984

14. Ford, L.R.; Fulkerson, D.R. A simple algorithm for finding maximal network flows and an application to the Hitchcock problem. Can. J. Math. 9:210-218; 1957.
15. Klein. M. A primal method for minimal cost flows with application to the assignment and transportation problems. Manag. Sci. 14:205-220: 1967.

16. Brok, M.; Slump, C.H. Automatic determination of image quality parameters in digital radiographic imaging systems. In: Schneider R.H.: Dwyer III, S.J.; Jost, R.G., eds. SPIE vol. 1090. Medical Imaging III: Image Formation; 1989:246-256.

17. Papoulis. A. Probability, random variables and stochastic processes. NY: McGraw Hill; 1965.

18. Wagner, R.F. Toward a unified view of radiological imaging systems, part II: noisy images. Med. Phys. 4:279-298; 1977.

19. Barnes, G.T. Radiographic mottle: a comprehensive theory. Med Phys. 9:656-667; 1982.

20. Domain 3D Graphics Metafile Resource Call Reference. HP/ Apollo, Palo Alto; 1989

21. Verhoeven, L.A.J. Digital subtraction angiography. Ph.D Thesis. Technical University Delft, the Netherlands, 1985.

22. Phong. B.T. Illumination for computer generated pictures. Comm. ACM 18:311-317: 1975 .

About the Author-JoHANNES G. M. vaN DEN BROEK received the M.Sc. degree in electrical engineering from the University of Twente in 1991. He has worked since in industry in the development of hardware and software. especially for the control of manufacturing machines.

About the Author-CORNELIS H. SLUMP received the M.Sc. degree in electrical engineering from Delft University of Technology in 1979 and his Ph.D. degree from Rijksuniversiteit Groningen in 1984. In 1983 he went to Philips Medical Systems, where he has worked in the development of the all-digital cath. lab. (DCI). In 1989 he joined the electrical engineering department of University of Twente, where his research interests are digital signal processing and medical image processing.

About the Author-CORSTIAAN J. STORM MD is senior cardiologist working partly in the Zuiderziekenhuis in Rotterdam as head of the Department of Invasive and Non Invasive Cardiology and partly as visiting cardiologist in the University Hospital Leiden. Department of Invasive Cardiology with special interest in digital imaging in collaboration with the University of Twente.

About the Author-Ad C. VAN BENTHEM B.Sc. is working for Philips Medical Systems, Department Digital Radiology, as application specialist and consultant at the Leiden University Hospital. Department Invasive Cardiology and Radiology.

About the Author-BEERT BUIS MD, Ph.D. is head of the Department Invasive Cardiology, Leiden University Hospital. 